\title{
Diagnostic advances
}

\section{Rapid detection of the major deletion in the Batten disease gene CLN3 by allele specific PCR}

\author{
Peter E M Taschner, Nanneke de Vos, Martijn H Breuning
}

\begin{abstract}
The recent isolation of the CLN3 gene involved in Batten disease (juvenile neuronal ceroid lipofuscinosis) creates possibilities for direct detection of mutations which can confirm or indicate the clinical diagnosis of Batten disease. We have designed a rapid and reliable allele specific PCR test for the detection of the major deletion, which can be used in carrier diagnosis, presymptomatic diagnosis, and prenatal diagnosis.

(F Med Genet 1997;34:955-956)
\end{abstract}

Keywords: neuronal ceroid lipofuscinosis; Batten disease; NCL; CLN3

Batten disease (juvenile neuronal ceroid lipofuscinosis) (MIM 204200) is an autosomal recessive neurodegenerative disorder characterised by progressive visual failure, seizures, and dementia. The presence of fingerprint patterns in the lysosomes of different tissues and the finding of vacuolated lymphocytes confirms the clinical diagnosis. ${ }^{1}$ The biochemical defect causing Batten disease still remains unknown.

Recently, the CLN3 gene which is involved in Batten disease was isolated using positional cloning methods. ${ }^{2}$ The D16S299-D16S298 haplotype 5,6 is shared by $73 \%$ of the Batten disease chromosomes, suggesting a founder effect. A patient homozygous for this major haplotype carried a $1.02 \mathrm{~kb}$ genomic deletion, encompassing exons 7 and 8 . This deletion was subsequently found on all 5,6 chromosomes. ${ }^{2}$ Direct detection of the major deletion by PCR would greatly improve the reliability of carrier diagnosis, which was previously performed by haplotype analysis. ${ }^{3}$ However, the use of PCR primers F2 and P3 which flank the deletion often results in preferential amplification of the deletion allele over the wild type because of the large difference in size between the products. ${ }^{4}$ This may lead to false test results. Therefore, we have designed a new PCR based test using a primer spanning the major deletion, in combination with one primer within the deletion and one primer outside the deletion (fig 1A). The test allows the simultaneous detection of normal and major deletion alleles and is non-radioactive, in contrast to a recently developed diagnostic test based upon solid phase minisequencing. ${ }^{5}$

We carried out the PCR test on $50 \mathrm{ng}$ genomic DNA in a total volume of $25 \mu \mathrm{l}$ at a final concentration of $50 \mathrm{mmol} / 1 \mathrm{KCl}, 1.5$ $\mathrm{mmol} / \mathrm{l} \mathrm{MgCl}_{2}, 200 \mu \mathrm{mol} / 1$ each $\mathrm{dNTP}, 0.004$ $\mathrm{U} / \mu \mathrm{l}$ of SuperTaq (HT Biotechnology Ltd, Cambridge, UK), in the presence of $5 \mathrm{pmol}$ of primers 2.3LR3 (5'-GGGGGAGGACAAG CACTG-3') and 2.3IntF7 (5'-CATTCTGT CACCCTTAGAAGCC-3') and 4 pmol of primer CLN3mut756R (5'-GGACTTGAAG GACGGAGTCT-3'). Primers were designed using the genomic CLN3 sequence (Genbank Acc No X99832). ${ }^{6}$ We have adjusted the primer concentrations to compensate for the more

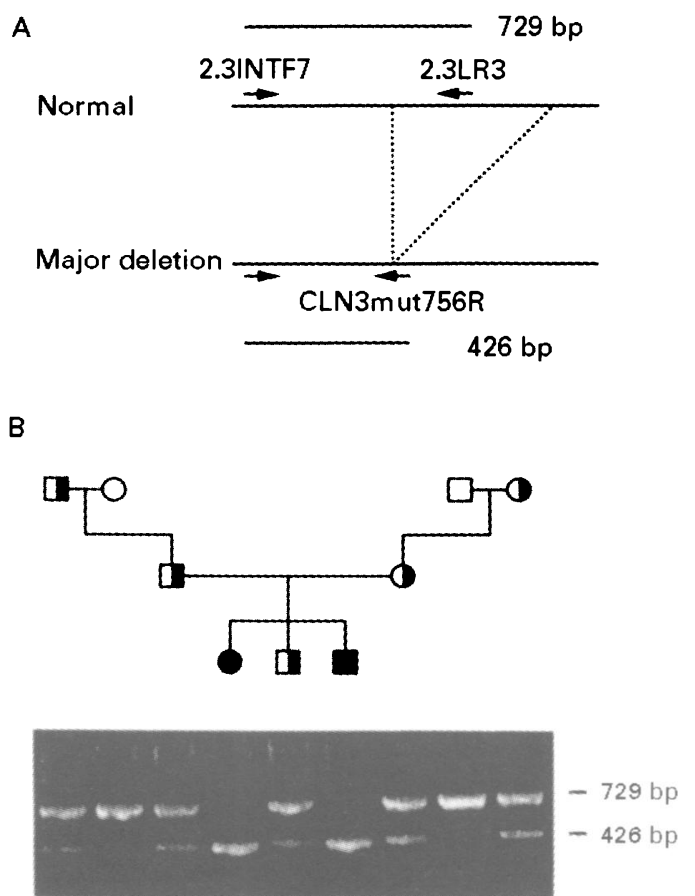

Figure 1 (A) Schematic representation of the normal and 5,6 deletion alleles of $C L N 3$. The annealing site of primer $2.3 L R 3$ is located within the deleted region whereas primer $C L N 3 m u t 756 R$ spans the deletion junction. The PCR products obtained by amplification of each allele are indicated. $(B)$ Direct detection of the major deletion in the family of a Batten patient. The patient (black symbol) has the $426 \mathrm{bp}$ major deletion fragment, normal subjects (open symbols) have the 726 bp fragment, and carriers (half filled symbols) have both. 
efficient amplification of the deletion allele. Denaturation was for three minutes at $94^{\circ} \mathrm{C}$, followed by 35 cycles of amplification with denaturation for one minute at $94^{\circ} \mathrm{C}$, annealing for two minutes at $56^{\circ} \mathrm{C}$, and extension for one minute at $72^{\circ} \mathrm{C}$, with a final extension for 10 minutes. Twenty $\mu \mathrm{l}$ of PCR sample were analysed on a $2 \%$ agarose gel.

We analysed the previously haplotyped three generation family of a Batten patient homozygous for the D16S299-D16S298 haplotype 5,6 using the allele specific PCR test (fig 1B). Two normal grandparents and all carriers of haplotype 5,6 share the 729 bp wild type PCR product. In addition, carriers have the 426 bp deletion PCR product, which is the only product observed from the Batten patient homozygous for haplotype 5,6 and thus for the major deletion. Therefore, the carrier status of all subjects corresponds to that determined by haplotype analysis, confirming the segregation of the major deletion with haplotype 5,6. Subsequently, we have used genomic DNA samples from 358 persons from 53 independent Batten families and 40 control samples with known D16S299-D16S298 haplotypes to evaluate the test. All samples from Batten families, except two, gave results which were consistent with their haplotypes and with segregation of their haplotypes within the families (data not shown). The two exceptions were carriers in which the wild type allele had not been sufficiently amplified. PCR products corresponding to both alleles were observed in subsequent tests of the same samples. The major deletion was not carried by control subjects.

New Batten patients and their families are now routinely screened for the presence of the major deletion. Out of nine new cases, six patients were homozygous for the major deletion, two patients were heterozygous, and the last one did not have the deletion (data not shown).

The clinical variability and the variations in the lipopigment patterns can delay reaching an accurate diagnosis, which is important for correct prognosis and genetic counselling. Direct detection of disease causing mutations in such complex disorders as Batten disease can accelerate reaching the correct diagnosis and may help to prevent the birth of additional patients. The allele specific PCR test described here has been applied with success to the confirmation of the clinical diagnosis in Batten patients and to the identification of carriers within their families, and can be used for prenatal diagnosis. This non-radioactive test is as fast as the radioactive solid phase minisequencing test. ${ }^{5}$ In contrast to haplotype based carrier detection, these tests can also be used to detect carriers of the major deletion in the general population, which is important for unrelated partners of proven carriers.

We thank the patients, their families, and the collaborating physicians for their cooperation. This research was supported by the National Institutes of Health, USA (NIH grant NS30152).

1 Brod RD, Packer AJ, Van Dyk HJL. Diagnosis of neuronal ceroid lipofuscinosis by ultrastructural examination of cripheral blood lymphocytes. Arch Ophthalmol 1987;105:1388-93.

2 The International Batten Disease Consortium. Isolation of a novel gene underlying Batten disease, CLN3. Cell 1995;82: 949-57.

3 Taschner PEM, de Vos N, Post JG, et al. Carrier detection of Batten disease (juvenile neuronal ceroid lipofuscinosis) Batten disease (juvenile neuron
Am $\mathcal{F}$ Med Genet 1995;57:333-7.

Am $\mathcal{F}$ Med Genet 1995;57:333-7.
Munroe PB, Rapola J, Mitchison HM, et al. Prenatal diagnosis of Batten's disease. Lancet 1996;347:1014-15.

5 Järvelä I, Mitchison HM, Munroe PB, et al. Rapid diagnostic test for the major mutation underlying Batten disease. $f$ Med Genet 1996;33:1041-2.

6 Mitchison HM, Munroe PB, O'Rawe AM, et al. Genomic structure and complete nucleotide sequence of the Batten disease gene, CLN3. Genomics 1997;40:346-50. 